\title{
A NONLINEAR SECOND ORDER PROBLEM WITH A NONLOCAL BOUNDARY CONDITION
}

\author{
P. AMSTER AND P. DE NÁPOLI
}

Received 28 January 2005; Accepted 28 March 2005

We study a nonlinear problem of pendulum-type for a $p$-Laplacian with nonlinear periodic-type boundary conditions. Using an extension of Mawhin's continuation theorem for nonlinear operators, we prove the existence of a solution under a LandesmanLazer type condition. Moreover, using the method of upper and lower solutions, we generalize a celebrated result by Castro for the classical pendulum equation.

Copyright (c) 2006 Hindawi Publishing Corporation. All rights reserved.

\section{Introduction}

The existence of periodic solutions for nonlinear second order ordinary differential equations have been widely studied, using the different techniques from nonlinear analysis, such as variational methods, topological methods and the method of upper and lower solutions.

In this work we consider an extension of the periodic problem for a nonlinear partial differential equation, namely:

$$
\begin{gathered}
\Delta_{p} u+g(u)=f(x) \quad \text { in } \Omega \\
u=c \quad \text { on } \partial \Omega \\
\int_{\partial \Omega}|\nabla u|^{p-2} \frac{\partial u}{\partial \eta}=h(c) .
\end{gathered}
$$

Here $c$ is a constant (whose value is unknown), $\Omega \subset \mathbb{R}^{N}$ is a bounded domain with smooth boundary, and $\Delta_{p}$ is the $p$-Laplacian $(1<p<\infty)$ :

$$
\Delta_{p} u=\operatorname{div}\left(|\nabla u|^{p-2} \nabla u\right)
$$

The boundary integral condition

$$
\int_{\partial \Omega}|\nabla u|^{p-2} \frac{\partial u}{\partial \eta}=h(c)
$$

Hindawi Publishing Corporation

Abstract and Applied Analysis

Volume 2006, Article ID 38532, Pages 1-11

DOI 10.1155/AAA/2006/38532 
will be understood in the sense, which holds for smooth functions, that

$$
\int_{\Omega} \Delta_{p} u=h(c)
$$

Some physical motivation for the study of this kind of problems (with $p=2$ ) comes from [2], where the authors study a model describing the equilibrium of a plasma confined in a toroidal cavity. Under appropriate conditions this model can be reduced to the nonhomogeneous boundary-value problem

$$
\begin{gathered}
\Delta u+h(x, u)=0 \quad \text { in } \Omega \\
\left.u\right|_{\partial \Omega}=\text { constant } \\
-\int_{\partial \Omega} \frac{\partial u}{\partial v}=I .
\end{gathered}
$$

The authors prove the existence of at least one solution $u \in H^{2}$ of the problem for any $h$ satisfying the following assumptions:

(A1) $h: \bar{\Omega} \times \mathbb{R} \rightarrow[0,+\infty)$ is continuous, nondecreasing on $u$, with $h(x, u)=0$ for $u \leq$ 0 .

(A2) $\lim _{u \rightarrow+\infty} \int_{\Omega} h(x, u) d x>I$.

(A3) $\lim _{u \rightarrow+\infty}(h(x, u)) / u^{r}=0$ for some $r \in \mathbb{R}$ (with $r \leq n /(n-2)$ when $n>2$ ).

In this work, we will show that some of the techniques that have been proved to be useful for the study of periodic solutions of ordinary differential equations can be applied to problem (1.1). For related results for Dirichlet boundary conditions, see, for example, [5].

First we consider the case in which $g, h: \mathbb{R} \rightarrow \mathbb{R}$ are continuous and $T$-periodic functions such that

$$
\int_{0}^{T} g(t) d t=\int_{0}^{T} h(t) d t=0 .
$$

Under these assumptions, the following theorem can be proved by an application of the variational method.

Theorem 1.1. Let (1.6) hold, and assume that $f \in L^{p^{\prime}}(\Omega)$ satisfy $\bar{f}=0$, where

$$
\bar{f}:=\frac{1}{|\Omega|} \int_{\Omega} f
$$

Then there exists at least one weak solution $u \in W_{0}^{1, p}(\Omega)+\mathbb{R}$ of problem (1.1).

On the other hand, we will apply the method of upper and lower solutions in order to study problem (1.1). 
Definition 1.2. We call $\alpha \in C^{1}(\bar{\Omega})$ a lower solution of (1.1) if

$$
\begin{gathered}
\Delta_{p} \alpha+g(\alpha) \geq f(x) \quad \text { in } \Omega \\
\alpha=c_{\alpha} \quad \text { on } \partial \Omega \\
\int_{\partial \Omega}|\nabla \alpha|^{p-2} \frac{\partial \alpha}{\partial \eta} \leq h\left(c_{\alpha}\right)
\end{gathered}
$$

and $\beta \in C^{1}(\bar{\Omega})$ an upper solution of $(1.1)$ if

$$
\begin{gathered}
\Delta_{p} \beta+g(\beta) \leq f(x) \quad \text { in } \Omega \\
\beta=c_{\beta} \quad \text { on } \partial \Omega \\
\int_{\partial \Omega}|\nabla \beta|^{p-2} \frac{\partial \beta}{\partial \eta} \geq h\left(c_{\beta}\right),
\end{gathered}
$$

where the inequalities are understood in the weak sense. Then we have the following theorem (for a related result for the case $h \equiv 0$, see [10]).

Theorem 1.3. Let $\alpha, \beta \in C^{1}(\bar{\Omega}), \alpha \leq \beta$ be a lower solution and an upper solution as above, and assume that $f \in L^{\infty}(\Omega)$. Then there exists a solution $u \in C^{1, r}(\bar{\Omega})$ for some $r \in(0,1)$ of problem (1.1) such that $\alpha \leq u \leq \beta$.

In particular, we may apply this result to an $n$-dimensional pendulum-like equation with nonlinear boundary conditions: assume that $g$ and $h$ are $T$-periodic, and let $f \in$ $L^{\infty}(\Omega)$ be fixed. Consider the set

$$
\mathscr{C}=\left\{\left(c_{1}, c_{2}\right) \in \mathbb{R}^{2}:(1.11) \text { is solvable }\right\},
$$

where problem (1.11) is defined by

$$
\begin{gathered}
\Delta_{p} u+g(u)=f(x)+c_{1} \quad \text { in } \Omega \\
u=c \quad \text { on } \partial \Omega \\
\int_{\partial \Omega}|\nabla u|^{p-2} \frac{\partial u}{\partial \eta}=h(c)-c_{2} .
\end{gathered}
$$

The following result can be regarded as an extension of the well known result obtained by Castro in [3] for the classical pendulum equation, and related results in $[1,7]$.

THEOREM 1.4. With the previous notations, assume that (1.6) hold, and that $\left(\underline{c_{1}}, \underline{c_{2}}\right),\left(\overline{c_{1}}, \overline{c_{2}}\right)$ $\in \mathscr{C}$, with

$$
\underline{c_{1}} \leq \overline{c_{1}}, \quad \underline{c_{2}} \leq \overline{c_{2}} .
$$

Then $\left(c_{1}, c_{2}\right) \in \mathscr{C}$ for any $\left(c_{1}, c_{2}\right)$ such that

$$
\underline{c_{1}} \leq c_{1} \leq \overline{c_{1}}, \quad \underline{c_{2}} \leq c_{2} \leq \overline{c_{2}} .
$$

Finally, we consider the case in which $g$ is a nonperiodic bounded function. More precisely, we have the following theorem, which asserts the existence of solutions under conditions of Landesman-Lazer type [9]. 
Theorem 1.5. Assume that $f \in L^{\infty}(\Omega)$, and $g$ is bounded. Further, assume that

$$
\limsup _{s \rightarrow+\infty} g(s)+\limsup _{s \rightarrow+\infty} \frac{h(s)}{|\Omega|}<\bar{f}<\liminf _{s \rightarrow-\infty} g(s)+\liminf _{s \rightarrow-\infty} \frac{h(s)}{|\Omega|}
$$

or

$$
\limsup _{s \rightarrow-\infty} g(s)+\limsup _{s \rightarrow-\infty} \frac{h(s)}{|\Omega|}<\bar{f}<\liminf _{s \rightarrow+\infty} g(s)+\liminf _{s \rightarrow+\infty} \frac{h(s)}{|\Omega|} .
$$

Then problem (1.1) admits at least one solution $u \in C^{1, r}(\bar{\Omega})$ for some $r>0$.

The proof of this theorem is based on a generalization of Mawhin coincidence degree theory for the case of quasi-linear operators. This generalization goes back to a paper by Manásevich and Mawhin [11], and was formulated in abstract form by Ge and Ren in $[8]$.

It is worth to note that, as in the classical Landesman-Lazer result, if

$$
\begin{gathered}
\limsup _{s \rightarrow-\infty} g(s)<g(x)<\underset{s \rightarrow+\infty}{\limsup } g(s), \\
\limsup _{s \rightarrow-\infty} h(s)<h(x)<\limsup _{s \rightarrow+\infty} h(s)
\end{gathered}
$$

then condition (1.14) is also necessary. This result follows immediately by integrating the equation. As it was shown in [6], a different situation occurs under Dirichlet conditions, where Landesman-Lazer conditions are no longer necessary.

Finally, we remark that the nonlinear character of the $p$-Laplacian when $p \neq 2$, introduces many differences with the case of the ordinary Laplacian considered in [1]. For instance, we prove a comparison principle suitable for the nonlinear case (Lemma 3.1), and we use the quasilinear version of the coincidence degree theory. Moreover, the optimal regularity of the solutions is different from the linear case. In general, it can only asserted that they are $C^{1, r}(\Omega)$, for some $r \in(0,1)$, and not in $W^{2, p}(\Omega)$ when $p \neq 2$.

This paper is organized as follows. In Section 2, we prove Theorem 1.1. In Section 3, we prove Theorems 1.3 and 1.4. Finally, in Section 4, we prove Theorem 1.5.

\section{Existence by variational methods}

For a proof of Theorem 1.1, let us consider the following functional in the space $W_{0}^{1, p}(\Omega)+\mathbb{R}:$

$$
I(u)=\int_{\Omega}\left(\frac{|\nabla u|^{p}}{p}-G(u)+f u\right)-H\left(\left.u\right|_{\partial \Omega}\right),
$$

where $G(s)=\int_{0}^{s} g(t) d t$ and $H(s)=\int_{0}^{s} h(t) d t$.

Lemma 2.1. Let $\Omega \subset \mathbb{R}^{N}$ a smooth bounded domain, and let

$$
\varphi_{n}(x)= \begin{cases}n \cdot d(x, \partial \Omega) & \text { if } d(x, \partial \Omega) \leq 1 / n \\ 1 & \text { if } d(x, \partial \Omega)>1 / n\end{cases}
$$


Then $\varphi_{n} \in W^{1, \infty}(\Omega)$ and if $v \in C\left(\bar{\Omega}, \mathbb{R}^{N}\right)$ then

$$
-\int_{\Omega} v \cdot \nabla \varphi_{n} \longrightarrow \int_{\partial \Omega} v \cdot \eta
$$

Proof. For $v \in C^{1}\left(\bar{\Omega}, \mathbb{R}^{N}\right)$, the result is immediate by the divergence theorem, and the general case follows by density.

LEMmA 2.2. If $u$ is a critical point of $I$ in the space $W_{0}^{1, p}(\Omega)+\mathbb{R}$, then $u$ is a weak solution of problem (1.1).

Proof. The derivative of $I$ is given by:

$$
\left\langle I^{\prime}(u), \varphi\right\rangle=\int_{\Omega}|\nabla u|^{p-2} \nabla u \nabla \varphi-\int_{\Omega} g(u) \varphi+\int_{\Omega} f(x) \varphi-\left.h\left(\left.u\right|_{\partial \Omega}\right) \varphi\right|_{\partial \Omega}
$$

for all $\varphi \in W_{0}^{1, p}(\Omega)+\mathbb{R}$ Hence, if $u$ is a critical point of $I, u$ is a weak solution of

$$
\Delta_{p} u+g(u)=f(x)
$$

From regularity theory $[4,14]$, it follows that $u \in C^{1, r}(\bar{\Omega})$ for some $r>0$. Furthermore, (by choosing $\varphi \equiv 1$ ) we see that

$$
\int_{\Omega} g(u)=\int_{\Omega} f(x)-h\left(\left.u\right|_{\partial \Omega}\right)
$$

On the other hand, by choosing $\varphi=\varphi_{n}$ as in Lemma 2.1 and letting $n \rightarrow \infty$ we have that:

$$
\int_{\partial \Omega}|\nabla u|^{p-2} \frac{\partial u}{\partial \eta}=\int_{\Omega}[g(u)-f(x)]
$$

Hence, we conclude that $u$ solves the weak formulation of problem (1.1).

Proof of Theorem 1.1. It is well known that the functional $I$ is weakly lower semicontinuous on $W_{0}^{1, p}(\Omega)+\mathbb{R}$, and bounded from below. In order to show that $I$ achieves a minimum, let us consider a minimizing sequence $\left\{u_{n}\right\} \subset W_{0}^{1, p}(\Omega)+\mathbb{R}$. We observe that since $G$ and $H$ are $T$-periodic then

$$
I(u+T)=I(u) \quad \forall u \in W_{0}^{1, p}(\Omega)+\mathbb{R} .
$$

Hence, we may assume that $\left.u_{n}\right|_{\partial \Omega} \in[0, T]$ for every $n$. From Poincaré inequality,

$$
\left\|u_{n}-\left.u_{n}\right|_{\partial \Omega}\right\|_{L^{p}}^{p} \leq c|| \nabla u_{n}\left\|_{L^{p}}^{p} \leq c_{1} I\left(u_{n}\right)+c_{2}\right\| u_{n} \|_{L^{p}}+c_{3},
$$

and it follows that $\left\{u_{n}\right\}$ is bounded in $W_{0}^{1, p}(\Omega)+\mathbb{R}$. By standard results, $I$ achieves a minimum, and the proof is complete. 
6 A nonlinear second order problem

\section{The method of upper and lower solutions}

In order to apply the method of upper and lower solutions to our problem, we will first prove an associated comparison principle.

Lemma 3.1. Let $\lambda>0, \rho: \mathbb{R} \rightarrow \mathbb{R}$ a continuous nondecreasing function, and let $\Omega_{1}$ be an open subset of $\Omega$. Assume that $u, v \in C_{0}^{1, r}(\bar{\Omega})+\mathbb{R}$ satisfy:

$$
\Delta_{p} u-\lambda|u|^{p-2} u \geq \Delta_{p} v-\lambda|v|^{p-2} v \quad \text { in } \Omega-\overline{\Omega_{1}}
$$

in weak sense,

$$
\begin{gathered}
\int_{\partial \Omega}|\nabla u|^{p-2} \frac{\partial u}{\partial \nu}+\rho\left(\left.u\right|_{\partial \Omega}\right) \leq \int_{\partial \Omega}|\nabla v|^{p-2} \frac{\partial v}{\partial \nu}+\rho(v \mid \partial \Omega) \\
u \leq v \quad \text { on } \partial \Omega_{1} .
\end{gathered}
$$

Then $u \leq v$ in $\Omega-\Omega_{1}$.

Proof. Let us consider the positive test function $\left.(u-v)^{+}\right|_{\Omega-\overline{\Omega_{1}}}$ and

$$
\Omega^{+}=\left\{x \in \Omega-\Omega_{1}: u(x)>v(x)\right\} .
$$

Then

$$
\begin{aligned}
\int_{\Omega^{+}} & \left(|\nabla u|^{p-2} \nabla u-|\nabla v|^{p-2} \nabla v\right) \nabla(u-v)+\lambda \int_{\Omega^{+}}\left(|u|^{p-2} u-|v|^{p-2} v\right)(u-v) \\
& \leq\left(\left.u\right|_{\partial \Omega}-\left.v\right|_{\partial \Omega}\right)^{+} \int_{\partial \Omega}\left(|\nabla u|^{p-2} \frac{\partial u}{\partial \eta}-|\nabla v|^{p-2} \frac{\partial v}{\partial \eta}\right) \\
& \leq\left(\left.u\right|_{\partial \Omega}-\left.v\right|_{\partial \Omega}\right)^{+}\left(\rho\left(\left.v\right|_{\partial \Omega}\right)-\rho\left(\left.u\right|_{\partial \Omega}\right)\right) \leq 0 .
\end{aligned}
$$

On the other hand, from strict monotonicity of the $p$-Laplacian, if $\left|\Omega^{+}\right|>0$ then

$$
\int_{\Omega^{+}}\left(|\nabla u|^{p-2} \nabla u-|\nabla v|^{p-2} \nabla v\right) \cdot \nabla(u-v)>0
$$

a contradiction.

Lemma 3.2. Let $\lambda, \mu>0$. Then, for every $\phi \in L^{p^{\prime}}(\Omega)$ and $k \in \mathbb{R}$ the problem

$$
\begin{gathered}
\Delta_{p} u-\lambda|u|^{p-2} u=\phi \quad \text { in } \Omega \\
u=c \quad \text { on } \partial \Omega \\
\int_{\partial \Omega}|\nabla u|^{p-2} \frac{\partial u}{\partial \eta}+\left.\mu|c|\right|^{p-2} c=k
\end{gathered}
$$

has a unique solution. Moreover, the mapping $(\phi, k) \mapsto u$ from $L^{p^{\prime}}(\Omega) \times \mathbb{R}$ to $W_{0}^{1, p}(\Omega)+\mathbb{R}$ is compact. 
Proof. Let us consider the functional $I: W_{0}^{1, p}(\Omega)+\mathbb{R} \rightarrow \mathbb{R}$ given by:

$$
I(u)=\int_{\Omega}\left(\frac{|\nabla u|^{p}}{p}+\lambda \frac{|u|^{p}}{p}+\phi u\right)-\left(\left.k u\right|_{\partial \Omega}-\left.\mu|u|_{\partial \Omega}\right|^{p}\right) .
$$

As before, it is easy to see that any critical point of $I$ is a solution of the problem. Moreover, $I$ is coercive, and the existence of a minimum of $I$ follows from standard results. Uniqueness follows from the comparison principle, and compactness follows from standard arguments (see, e.g., [5])

Proof of Theorem 1.3. Choose $\lambda, \mu>0$ and define the function $P$ given by:

$$
P(x, u)= \begin{cases}u & \text { if } \alpha(x) \leq u \leq \beta(x) \\ \beta(x) & \text { if } u>\beta(x) \\ \alpha(x) & \text { if } u<\alpha(x)\end{cases}
$$

and consider the following fixed point operator $T: W_{0}^{1, p}(\Omega)+\mathbb{R} \rightarrow W_{0}^{1, p}(\Omega)+\mathbb{R}$. For fixed $u$, define $T(u)$ as the unique solution $v$ of the problem:

$$
\begin{gathered}
\Delta_{p} v-\lambda|v|^{p-2} v=f(x)-g(P(x, u))-\lambda|P(x, u)|^{p-2} P(x, u) \\
\left.v\right|_{\partial \Omega}=c \\
\int_{\partial \Omega}|\nabla v|^{p-2} \frac{\partial v}{\partial \eta}+\mu|c|^{p-2} c=h(P(x, u) \mid \partial \Omega)+\mu|P(x, u)| \partial \Omega||^{p-2} P(x, u) \mid \partial \Omega .
\end{gathered}
$$

From Lemma 3.2, $T$ is well-defined and compact. As the right-hand side term is bounded, it follows from Schauder theorem that $T$ has a fixed point $u$. We claim that $\alpha \leq u \leq \beta$, and hence $u$ is a solution of the problem. Indeed, if we define $\Omega_{1}=\{x \in \Omega: u(x)<\beta(x)\}$ then for $x \in \Omega-\Omega_{1}$ it holds:

$$
\Delta_{p} u-\lambda|u|^{p-2} u=f(x)-g(\beta)-\lambda|\beta|^{p-2} \beta \geq \Delta_{p} \beta-\lambda|\beta|^{p-2} \beta
$$

and from the comparison principle it follows that $u \leq \beta$ in $\Omega-\Omega_{1}$. In the same way, it follows that $u \geq \alpha$. From regularity theory, it follows that $u \in C^{1, r}(\bar{\Omega})$ for some $r \in$ $(0,1)$.

Proof of Theorem 1.4. Let $\beta$ and $\alpha$ be solutions of (1.11) for $\left(\underline{c_{1}}, \underline{c_{2}}\right)$ and $\left(\overline{c_{1}}, \overline{c_{2}}\right)$ respectively. As $g$ is $T$-periodic and $\alpha, \beta \in C(\bar{\Omega})$, adding a term $k T(k \in \bar{N})$ if necessary, we may suppose that $\alpha \leq \beta$. From definition, it is clear that $\alpha$ is a lower solution and $\beta$ is an upper solution of (1.11) with $c_{1}$ and $c_{2}$, and the proof follows from Theorem 1.3.

\section{Coincidence degree methods}

In this section we recall a continuation theorem due to Ge and Ren [8], which extends a classical result by Mawhin [12]. For convenience, we follow the version in [13]. 
Let $X$ and $Z$ be Banach spaces with norms $\|\cdot\|_{X}$ and $\|\cdot\|_{Z}$, respectively. A continuous operator $M: X \cap \operatorname{dom} M \rightarrow Z$ is said to be quasi-linear if

(i) $\operatorname{Im} M=M(X \cap \operatorname{dom} M)$ is a closed subset of $Z$;

(ii) $\operatorname{Ker} M=\{x \in X \cap \operatorname{dom} M: M x=0\}$ is linearly homeomorphic to $\mathbb{R}^{n}, n<\infty$.

Let $P: X \rightarrow X_{1}$ and $Q: Z \rightarrow Z_{1}$ be two projectors such that $\operatorname{Im} P=\operatorname{Ker} M, \operatorname{Ker} Q=\operatorname{Im} M$ and $X=X_{1} \oplus X_{2}, Z=Z_{1} \oplus Z_{2}$, where $X_{1}=\operatorname{Ker} M, Z_{2}=\operatorname{Im} M$ and $X_{2}, Z_{1}$ are respectively the complement space of $X_{1}$ in $X, Z_{2}$ in $Z$. If $U$ is an open and bounded subset of $X$ such that $\operatorname{dom} M \cap U \neq \varnothing$, the continuous operator $N_{\lambda}: \bar{U} \rightarrow Z, \lambda \in[0,1]$ will be called $M$-compact in $\bar{U}$ with respect to $M$ if

(iii) There is a subset $Z_{1}$ of $Z$ with $\operatorname{dim} Z_{1}=\operatorname{dim} X_{1}$ and an operator $K: \operatorname{Im} M \rightarrow X_{2}$ with $K 0=0$ such that for $\lambda \in[0,1]$,

$$
\begin{gathered}
(I-Q) N_{\lambda}(\bar{U}) \subset \operatorname{Im} M \subset(I-Q) Z, \\
(I-Q) N_{0}=0, \quad Q N_{\lambda} x=0 \Longleftrightarrow Q N x=0, \quad \lambda \in(0,1), \\
K M=I-P, \quad K(I-Q) N_{\lambda}: \bar{U} \longrightarrow X_{2} \subset X \text { is compact, } \\
M\left[P+K(I-Q) N_{\lambda}\right]=(I-Q) N_{\lambda} .
\end{gathered}
$$

Theorem 4.1 ([8]). Let $X$ and $Z$ be two Banach spaces with the norms $\|\cdot\|_{X}$ and $\|\cdot\|_{Z}$, respectively, and $U \subset X$ an open and bounded set. Suppose $M: X \cap \operatorname{dom} M \rightarrow Z$ is a quasilinear operator and $N_{\lambda}: \bar{U} \rightarrow Z$ is $M$-compact with respect to $M$. In addition, if

(C1) $M x \neq N_{\lambda} x, \lambda \in(0,1), x \in \partial U$

(C2) $\operatorname{deg}(J Q N, U \cap \operatorname{Ker} M, 0) \neq 0$, where $N=N_{1}$ and $J: Z_{1} \rightarrow X_{1}$ is a homeomorphism with $J(0)=0$;

then the abstract equation $M x=N x$ has at least one solution in $\bar{U}$.

The proof of this continuation theorem is based on a Lyapunov-Schmidt reduction argument and an application of the Leray-Schauder degree theory.

In order to apply the continuation theorem to our problem, let us consider:

$$
\begin{gathered}
X=W_{0}^{1, p}(\Omega)+\mathbb{R}=\left\{u \in W^{1, p}(\Omega): u_{\mid \partial \Omega} \text { is constant }\right\} \\
Z=L^{p^{\prime}}(\Omega) \times \mathbb{R} \\
M(u)=\left(\Delta_{p} u, \int_{\partial \Omega} \Delta_{p} u\right) \\
\operatorname{dom}(M)=\left\{u \in X: \Delta_{p} u \in L^{p^{\prime}}(\Omega)\right\} .
\end{gathered}
$$

Then

$$
\begin{gathered}
\operatorname{Ker}(M)=\mathbb{R}, \\
\operatorname{Im}(M)=\left\{(f, c) \in Y: \int_{\Omega} f(x)=c\right\} .
\end{gathered}
$$

We may define the projectors $P$ and $Q$ as

$$
\begin{gathered}
P(u)=\bar{u} \\
Q(f, c)=\left(\bar{f}-\frac{c}{|\Omega|}, 0\right) .
\end{gathered}
$$


Then

$$
\operatorname{Im}(Q)=\{(c, 0): c \in \mathbb{R}\}
$$

and we may define $J(c, 0)=c$. For $(f, c) \in \operatorname{Im}(M)$, we define $K(f, c)$ as the unique solution of the problem

$$
\begin{gathered}
\Delta_{p} u=f(x) \quad \text { in } \Omega \\
u=c \quad \text { on } \partial \Omega \\
\bar{u}=0 .
\end{gathered}
$$

Finally, let us consider

$$
N(u)=\left(f-g(u), h\left(u_{\mid \partial \Omega}\right)\right) .
$$

It follows from the strong monotonicity of $M$ that $K(I-Q) N_{\lambda}$ is compact on $U$ for any open bounded subset $U \subset X$.

\subsection{A priori bounds}

Proposition 4.2. Let us assume that the conditions of Theorem 1.5 hold. Then there exists a constant $R_{0}>0$ such that if $u$ is a solution of

$$
\begin{gathered}
\Delta_{p} u=\lambda[f(x)-g(u)] \quad \text { in } \Omega \\
u=c \quad \text { on } \partial \Omega \\
\int_{\partial \Omega}|\nabla u|^{p-2} \frac{\partial u}{\partial \eta}=\lambda h(c)
\end{gathered}
$$

with $\lambda \in(0,1]$, then $\|u\|_{W^{1, p}} \leq R_{0}$.

Proof. It suffices to consider only the case in which (1.14) holds, since the other case is similar. Assume by contradiction that we have a sequence $\left(u_{n}\right)$ of solutions of

$$
\begin{gathered}
\Delta_{p} u_{n}=\lambda_{n}[f(x)-g(u)] \quad \text { in } \Omega \\
u_{n}=c_{n} \quad \text { on } \partial \Omega \\
\int_{\partial \Omega}\left|\nabla u_{n}\right|^{p-2} \frac{\partial u_{n}}{\partial \eta}=\lambda_{n} h\left(c_{n}\right)
\end{gathered}
$$

such that $\left\|u_{n}\right\|_{W^{1, p}} \rightarrow+\infty$. Set $v_{n}=u_{n}-c_{n}$. Then we have that

$$
\begin{gathered}
\Delta_{p} v_{n}=\lambda_{n}\left[f(x)-g\left(v_{n}+c_{n}\right)\right] \quad \text { in } \Omega \\
v_{n}=0 \text { on } \partial \Omega \\
\int_{\partial \Omega}\left|\nabla v_{n}\right|^{p-2} \frac{\partial v_{n}}{\partial \eta}=\lambda_{n} h\left(c_{n}\right) .
\end{gathered}
$$

As $g$ is bounded, we obtain:

$$
\left\|v_{n}\right\|_{W^{1, p}} \leq k_{1}\left\|\lambda_{n}\left(f(x)-g\left(v_{n}+c_{n}\right)\right)\right\|_{L^{p^{\prime}}} \leq k_{2} .
$$

It follows that $c_{n}$ is unbounded, and taking a subsequence we may assume that $c_{n} \rightarrow+\infty$, or that $c_{n} \rightarrow-\infty$. 
Since the imbedding $W^{1, p}(\Omega) \hookrightarrow L^{p}(\Omega)$ is compact, we can extract a subsequence $v_{n_{k}}$ such that $v_{n_{k}} \rightarrow v$ for the $L^{p}$-norm and $v_{n_{k}}(x) \rightarrow v(x)$ a.e.

If $c_{n_{k}} \rightarrow+\infty$,

$$
\limsup _{k \rightarrow \infty} g\left(v_{n_{k}}(x)+c_{n_{k}}\right) \leq \limsup _{s \rightarrow+\infty} g(s)
$$

a.e. in $\Omega$. Thus,

$$
\frac{1}{|\Omega|} \int_{\Omega} \limsup _{k \rightarrow \infty} g\left(v_{n_{k}}(x)+c_{n_{k}}\right) \leq \limsup _{s \rightarrow+\infty} g(s)
$$

and from Fatou's lemma:

$$
\limsup _{k \rightarrow \infty} \frac{1}{|\Omega|} \int_{\Omega} g\left(v_{n_{k}}(x)+c_{n_{k}}\right) \leq \lim \sup _{s \rightarrow+\infty} g(s) .
$$

By Lemma 2.1:

$$
\int_{\partial \Omega}\left|\nabla u_{n_{k}}\right|^{p-2} \frac{\partial u_{n_{k}}}{\partial \eta}+\lambda_{n_{k}} \int_{\Omega} g\left(u_{n_{k}}\right)=\lambda_{n_{k}} \int_{\Omega} f(x) d x
$$

or

$$
\bar{f}=\frac{h\left(c_{n_{k}}\right)}{|\Omega|}+\frac{1}{|\Omega|} \int_{\Omega} g\left(v_{n_{k}}(x)+c_{n_{k}}\right) .
$$

Then

$$
\bar{f} \leq \limsup _{s \rightarrow+\infty} g(s)+\limsup _{s \rightarrow+\infty} \frac{h(s)}{|\Omega|},
$$

a contradiction. In a similar way, we see that if $c_{n} \rightarrow-\infty$, then

$$
\liminf _{s \rightarrow-\infty} g(s)+\liminf _{s \rightarrow-\infty} \frac{h(s)}{|\Omega|} \leq \bar{f}
$$

and the proof follows.

Proof of Theorem 1.5. As before, we assume that (1.14) holds. In order to prove Theorem 1.5 , let us consider the bounded open set

$$
U=\left\{u \in X:\|u\|_{W^{1, p}}<R\right\}
$$

for some $R>R_{0}$ large enough, where $R_{0}$ is the bound given by Proposition 4.2.

It remains to show that $d_{B}(J Q N, U \cap \operatorname{Ker}(L), 0)$ is well defined and different from zero.

Let $k: \mathbb{R} \rightarrow \mathbb{R}$ be defined by:

$$
k(t)=J Q N(t)=\bar{p}-g(t)-\frac{h(t)}{|\Omega|} .
$$

From condition (1.14)

$$
k(R)>0>k(-R)
$$

for $R>R_{0}$ large.

Then $U \cap \operatorname{Ker}(L)=(-R, R)$ and we conclude that $d_{B}(J Q N, U \cap \operatorname{Ker}(L), 0) \neq 0$. Hence, all the conditions of Theorem 4.1 are fulfilled and the proof is complete. 


\section{Acknowledgment}

The authors gratefully acknowledge the research support of Fundación Antorchas.

\section{References}

[1] P. Amster, P. De Nápoli, and M. C. Mariani, Existence of solutions to N-dimensional pendulumlike equations, Electronic Journal of Differential Equations 2004 (2004), no. 125, 1-8.

[2] H. Berestycki and H. Brézis, On a free boundary problem arising in plasma physics, Nonlinear Analysis 4 (1980), no. 3, 415-436.

[3] A. Castro, Periodic solutions of the forced pendulum equation, Differential Equations (Proc. Eighth Fall Conf., Oklahoma State Univ., Stillwater, Okla., 1979), Academic Press, New York, 1980, pp. 149-160.

[4] E. DiBenedetto, $C^{1+\alpha}$ local regularity of weak solutions of degenerate elliptic equations, Nonlinear Analysis 7 (1983), no. 8, 827-850.

[5] G. Dinca, P. Jebelean, and J. Mawhin, Variational and topological methods for Dirichlet problems with p-Laplacian, Portugaliae Mathematica. Nova Série 58 (2001), no. 3, 339-378.

[6] P. Drábek, P. Girg, and P. Takáč, Bounded perturbations of homogeneous quasilinear operators using bifurcations from infinity, Journal of Differential Equations 204 (2004), no. 2, 265-291.

[7] G. Fournier and J. Mawhin, On periodic solutions of forced pendulum-like equations, Journal of Differential Equations 60 (1985), no. 3, 381-395.

[8] W. Ge and J. Ren, An extension of Mawhin's continuation theorem and its application to boundary value problems with a p-Laplacian, Nonlinear Analysis 58 (2004), no. 3-4, 477-488.

[9] E. M. Landesman and A. C. Lazer, Nonlinear perturbations of linear elliptic boundary value problems at resonance, J. Math. Mechanics 19 (1970), 609-623.

[10] V. K. Le and K. Schmitt, Sub-supersolution theorems for quasilinear elliptic problems: A variational approach, Electronic Journal of Differential Equations 2004 (2004), no. 118, 1-7.

[11] R. Manásevich and J. Mawhin, Periodic solutions for nonlinear systems with p-Laplacian-like operators, Journal of Differential Equations 145 (1998), no. 2, 367-393.

[12] J. Mawhin, Topological Degree Methods in Nonlinear Boundary Value Problems, CBMS Regional Conference Series in Mathematics, vol. 40, American Mathematical Society, Rhode Island, 1979.

[13] X. Ni and W. Ge, Multi-point boundary-value problems for the p-Laplacian at resonance, Electronic Journal of Differential Equations 2003 (2003), no. 112, 1-7.

[14] P. Tolksdorf, Regularity for a more general class of quasilinear elliptic equations, Journal of Differential Equations 51 (1984), no. 1, 126-150.

P. Amster: Universidad de Buenos Aires, FCEyN, Departamento de Matemática,

Ciudad Universitaria, Pabellón I, (1428) Buenos Aires, CONICET (Consejo Nacional de Investigaciones Científicas y Técnicas), Argentina

E-mail address: pamster@dm.uba.ar

P. De Nápoli: Universidad de Buenos Aires, FCEyN, Departamento de Matemática, Ciudad Universitaria, Pabellón I, (1428) Buenos Aires, CONICET (Consejo Nacional de Investigaciones Científicas y Técnicas), Argentina

E-mail address: pdenapo@dm.uba.ar 


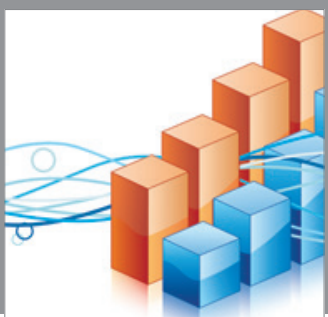

Advances in

Operations Research

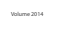

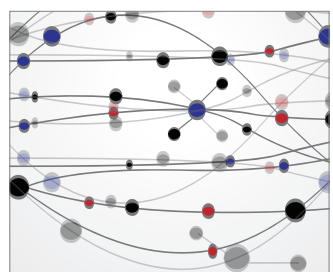

\section{The Scientific} World Journal
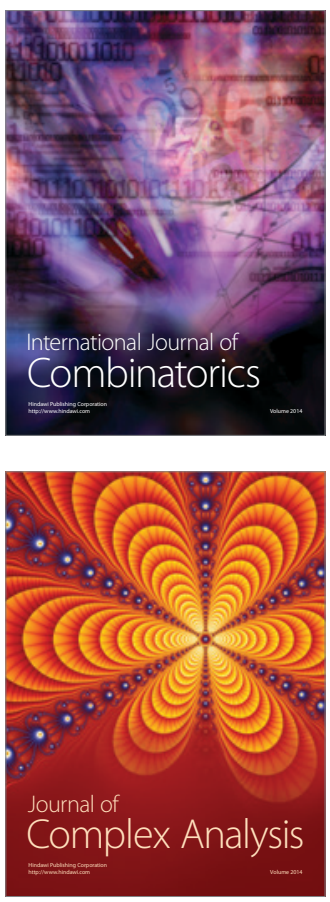

International Journal of

Mathematics and

Mathematical

Sciences
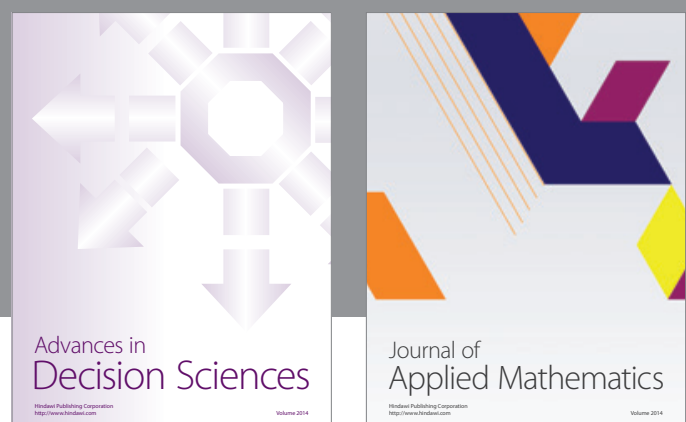

Journal of

Applied Mathematics
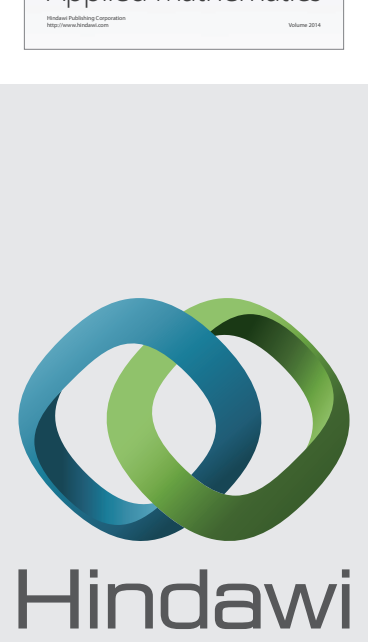

Submit your manuscripts at http://www.hindawi.com
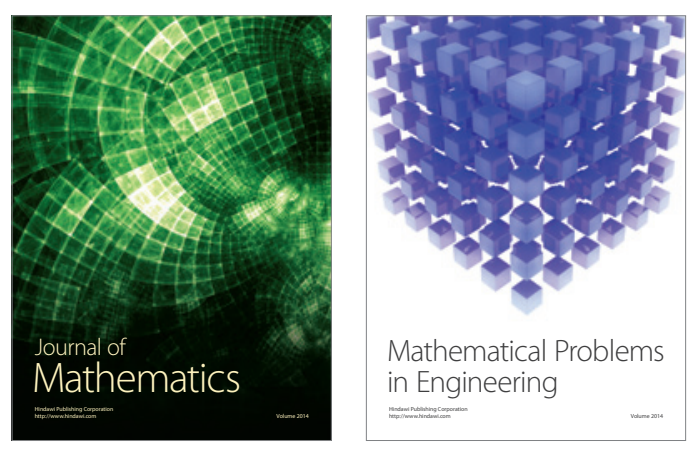

Mathematical Problems in Engineering
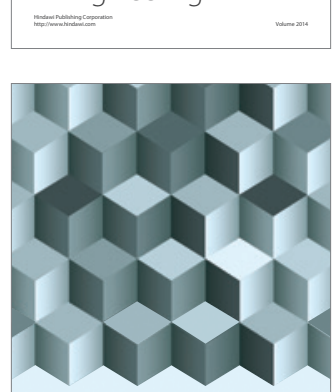

Journal of

Function Spaces
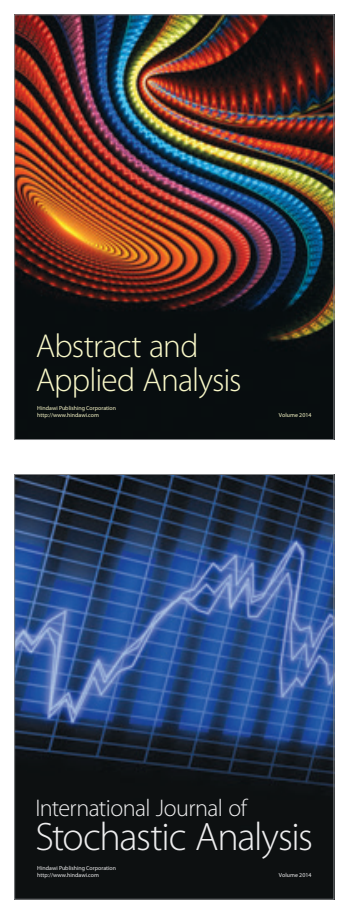

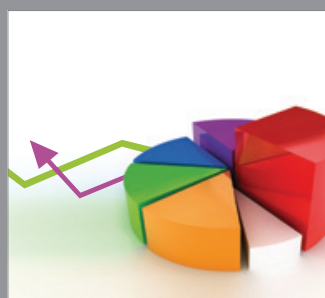

ournal of

Probability and Statistics

Promensencen
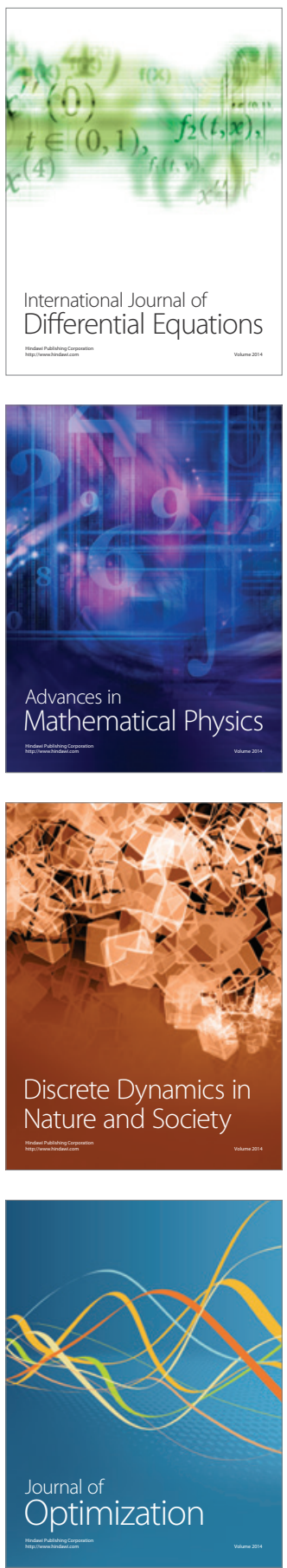